\title{
Infección por Bartonella henselae como presentación de fiebre de origen desconocida en niños peruanos
}

\section{Infection with bartonella henselae in patients with fever of unknown origin}

\section{Edwin Miranda-Choque, ${ }^{1}$ Jorge Candela-Herrera, ${ }^{1}$ Jenny Alvarado-Durand, ${ }^{1}$ María Esquivel-Quispe, ${ }^{1,3}$ Sonia Farfán-Ramos ${ }^{1 / 4}$}

1. Médico infectólogo. Instituto Nacional de Salud del Niño. Lima, Perú.

2. Médico pediatra. Hospital Nacional 2 de Mayo.

3. Licenciado en tecnología médica en la especialidad de Laboratorio clínico y anatomía patológica.

4. Enfermera especialista en pediatría. Instituto Nacional de Salud del Niño.

\section{RESUMEN}

Oвjetivo. Describir los casos por infección por Bartonella henselae como presentación de fiebre de origen desconocida (FOD) en el Instituto Nacional de Salud del Niño (INSN) de Perú. Material y métodos. Estudio de serie de casos de niños atendidos en el INSN en el año 2012. Se definió infección por B. henselae si el caso presentaba serología positiva. Resultados. En el año 2012 se diagnosticaron 26 casos de FOD, de los cuales 12 fueron por infección por B. henselae. De estos 12, 6 fueron masculinos, con una mediana para la edad de 5 años (RIQ 3,5-4,0); 11 tenían una historia de exposición a gatos; 6 tuvieron lesión lineal ocasionada por el gato. Dentro de los exámenes de laboratorio, 4 de 12 tuvieron leucocitosis; 5 presentaron una PCR mayor de 10. El examen ecográfico reveló que 8 de 12 presentaron lesiones hipoecoicas en bazo; existió coinfección con otras infecciones; 2 casos tuvieron manifestación articular y 1, convulsión. Conclusión. Se concluye que los casos de FOD por infección por B. henselae en niños, están relacionados con exposición a gatos y lesiones hipoecoicas en bazo e hígado, con una evolución de la enfermedad con pronóstico bueno.

Palabras clave. Fiebre de origen desconocido, Bartonella henselae, niños.

\section{SUMMARY}

Objective. To describe the cases of infection with Bartonella henselae as presentation of fever of unknown origin (FUO) at the National Institute of Child Health (NICH) of Peru. Material and Methods. Study of a series of cases of children cared for in the $\mathrm{NICH}$ in 2012, infection was defined by B. henselae if presented positive serology. Results. In 2012, 26 cases were diagnosed of FUO of which 12 were due to infection by $B$. henselae, 06 were male, with a median age of 5 years (IQR 3,5-4,0), 11 of 12 had a history of exposure to cats, 6 of 12 had linear lesion caused by the cat. Within the lab tests 4 out of 12 had leukocytosis, 5 of 12 presented a CRP of greater than 10. The ultrasound examination revealed that 8 out of 12 lesions were hypoechoics in the spleen; there was co-infection with other infections; 02 cases had demonstration articulate and one with seizure. We conclude that the cases of FUO by infection by B. henselae in children are related to school-age, with the exposure of cats and injury hypoechoic in the spleen. Conclusions. We conclude that the cases of FUO by infection by B. henselae in children are related to the exposure of cats and injury hypoechoic in the spleen and liver and good prognosis.

KEY WORDS. Fever of unknown origin, Bartonella henselae, child. 


\section{INTRODUCCIÓN}

La enfermedad por arañazo de gato (EAG) es una zoonosis ocasionada por Bartonella henselae, bacteria bacilo pleomórfica gramnegativa, de difícil aislamiento en sangre o en tejidos como hígado o bazo. Esta enfermedad es considerada de curso benigno y autolimitada. Los niños y los adultos jóvenes son los más afectados. ${ }^{1} \mathrm{Su}$ transmisión es más frecuente por inoculación en la piel a través de un arañazo, mordedura o lamido de gato. La presentación clínica se puede clasificar entre formas típicas y atípicas. La típica es la denominada adenitis regional, pero la forma atípica se basa en relación a la localización y el cuadro clínico como la presencia de fiebre. ${ }^{2}$

La B. henselae es considerada de cultivo difícil, por lo que el examen de laboratorio más usado es la inmunofluorescencia indirecta (IFI), que detecta anticuerpos de tipo inmunoglobulina $G(\operatorname{IgG})$ y de inmunoglobulina $M(\operatorname{IgM})$ contra $B$. henselae, con una sensibilidad de $88 \%$ y una especificidad de $94 \%$. Para el diagnóstico también puede emplearse la biopsia de tejido, mediante la tinción de Warthin-Starry de la muestra obtenida, pero es poco específica y de bajo rendimiento. ${ }^{3}$

Existen pocos reportes de EAG en Perú ${ }^{4}$ y son menos los relacionados con fiebre de origen desconocida (FOD), por lo que se decidió realizar el presente estudio, con el objetivo de describir la serie de casos de EAG como presentación de FOD en niños atendidos en el Instituto Nacional de Salud del Niño (INSN), hospital pediátrico de referencia nacional en Perú.

\section{MATERIAL Y MÉTODO}

Estudio de serie de casos. La población estuvo integrada por los pacientes atendidos en el INSN con diagnóstico de FOD y con exámenes serológicos para $B$. henselae, en el período enerodiciembre 2012, en los servicios de Infectología y Medicina. La información de los pacientes se obtuvo de las historias clínicas.

Se definió como caso de FOD al de los pacientes atendidos por fiebre de más de $38,3^{\circ} \mathrm{C}$ en varias ocasiones, de 14 días o más de duración sin causa conocida después de una semana de anamnesis, exploración y estudios complementarios hospita- larios o ambulatorios., ${ }^{5,6}$ Asimismo, se define infección por $B$. henselae a los casos con serología positiva a inmunoglobulinas IgG e IgM, mediante el método de $\mathrm{IFI}$, a una dilución de $1 / 100$ para IgM y de $1 / 320$ para la IgG, según el inserto 0 instrucciones. Para esto se usó un microscopio de fluorescencia marca Euroinmun (Alemania). El estudio fue aprobado por el comité del INSN.

\section{RESULTADOS}

Se estudió 12 casos de FOD y EAG, 6 de ellos fueron masculinos (50\%), con una mediana para la edad de 5 años (RIQ 3,3-7,5), todos con IgM e IgG reactivas. Ninguno de ellos presentaba comorbilidad de importancia, con respecto a la información epidemiológica relacionada al gato; 11 de 12 tenían una historia de exposición a gatos; en 10 de 12, el gato era la mascota de la familia. Tabla 1.

El tipo de lesión fue lineal en 6 de 12 casos, y se presentó pápula en 1 caso.

En cuanto a los exámenes de laboratorio, 4 de 12 tuvieron leucocitosis; 4 de 12, linfocitosis; 5 de 12, PCR mayor de 10; 5 de 12, hematócrito menos de $30 \% ; 1$ de 12, aminotransferasas elevadas. Tabla 2.

Tabla 1. Características epidemiológicas y manejo ambulatorio de los casos de fiebre de origen desconocida (FOD) por enfermedad de arañazo de gato (EAG)

Paciente Edad Fiebre (días) Sexo Ambulatorio

$\begin{array}{rrlll}\Delta 1 & 6 & 21 & M & \text { Sí } \\ \Delta 2 & 10 & 14 & F & \text { Sí } \\ \Delta 3 & 3 & 18 & \text { M } & \text { Sí } \\ \Delta 4 & 6 & 14 & F & \text { Sí } \\ \Delta 5 & 5 & 45 & F & \text { No } \\ \Delta 6 & 5 & 15 & \text { M } & \text { No } \\ \Delta 7 & 4 & 90 & F & \text { No } \\ \Delta 8 & 11 & 60 & F & \text { No } \\ \Delta 9 & 2 & 30 & \text { M } & \text { Sí } \\ \Delta 10 & 2 & 40 & F & \text { Sí } \\ \Delta 11 & 4 & 30 & \text { M } & \text { Sí } \\ \Delta 12 & 8 & 14 & \text { M } & \text { Sí }\end{array}$

M: masculino; f: femenino. 
Tabla 2. Características de la exposición al gato y el motivo de consulta de los casos de fiebre de origen desconocida (FOD) por enfermedad de arañazo de gato (EAG)

$\begin{array}{lllll}\text { Paciente } & & \text { Exposición al gato } & & \text { Motivo de consulta } \\ & \text { Reportado } & \text { Propietario } & \text { Lesión } & \\ \Delta 1 & \text { Sí } & \text { Propio } & \text { Lineal } & \text { Fiebre } \\ \Delta 2 & \text { Sí } & \text { Propio } & \text { Lineal } & \text { Fiebre } \\ \Delta 3 & \text { Sí } & \text { Propio } & \text { Escoriación } & \text { Fiebre, artralgia } \\ \Delta 4 & \text { Sí } & \text { Propio } & \text { Lineal } & \text { Fiebre } \\ \Delta 5 & \text { Sí } & \text { Propio } & \text { No lesión } & \text { Fiebre } \\ \Delta 6 & \text { Sí } & \text { Propio } & \text { Lineal, pápula } & \text { Fiebre, convulsión } \\ \Delta 7 & \text { Sí } & \text { Propio } & \text { No lesión } & \text { Fiebre, poliartralgia } \\ \Delta 8 & \text { No } & \text { Desconocido } & \text { No lesión } & \text { Fiebre } \\ \Delta 9 & \text { Sí } & \text { Propio } & \text { Lineal escoriación } & \text { Fiebre } \\ \Delta 10 & \text { Sí } & \text { Vecino } & \text { No lesión } & \text { Fiebre } \\ \Delta 12 & \text { Sí } & \text { Propio } & \text { Escoriación } & \text { Fiebre, dolor abdominal }\end{array}$

La evaluación ecográfica demostró 8 lesiones hipoecoicas en el bazo (8/12) y 5 en el hígado (5/8). Tabla 3.

Todos los pacientes fueron dados de alta con evolución buena.

\section{DISCUSIÓN}

La incidencia de FOD llega hasta $10 \%$ en otros países. ${ }^{1,7}$ En Perú, en el INSN, los casos de FOD por $B$. henselae son frecuentes $y$, según estudios realizados en el INSN, una de las primeras causas

Tabla 3. Características de la ecografía de abdomen de los casos de fiebre de origen desconocida (FOD) por enfermedad de arañazo de gato (EAG)

\begin{tabular}{llll} 
Paciente & \multicolumn{1}{c}{ Bazo } & \multicolumn{1}{c}{ Hígado } & \multicolumn{1}{c}{ Adenopatías } \\
$\Delta 1$ & Lesiones hipoecoicas múltiples & Normal & Mesentéricas \\
$\Delta 2$ & Normal & Normal & No \\
$\Delta 3$ & Lesiones hipoecoicas múltiples $(3 \mathrm{~mm})$ & Hepatomegalia & Mesentérica $(23 \mathrm{~mm})$ \\
$\Delta 4$ & Esplenomegalia & Hepatomegalia & Mesentéricas y retroperitoneales \\
& Lesiones hipoecoicas múltiples $(5 \mathrm{~mm})$ & Lesión única hipoecoica $(3 \mathrm{~mm})$ & múltiples $(8 \mathrm{~mm})$ \\
$\Delta 5$ & Esplenomegalia, lesiones & Hepatomegalia & Mesentéricas y retroperitoneales \\
& Lesiones hipoecoicas múltiples $(4 \mathrm{~mm})$ & Lesión única hipoecoica $(3 \mathrm{~mm})$ & múltiples $(7$ mm $)$ \\
$\Delta 6$ & Esplenomegalia & Normal & Retroperitoneales múltiples $(6 \mathrm{~mm})$ \\
$\Delta 8$ & Normal & Normal & No \\
$\Delta 9$ & Lesiones hipoecoicas múltiples $(3 \mathrm{~mm})$ & Hepatomegalia & Mesentéricas y retroperitoneales \\
$\Delta 10$ & Lesiones hipoecoicas múltiples & Lesiones hipoecoicas múltiples $(10 \mathrm{~mm})$ & múltiples $(8 \mathrm{~mm})$ \\
$\Delta 11$ & Lesiones hipoecoicas múltiples & Normal & Mesentérica \\
$\Delta 12$ & Lesiones hipoecoicas múltiples $(4 \mathrm{~mm})$ & Lesiones hipoecoicas múltiples $(7 \mathrm{~mm})$ & mesentérica $(4 \mathrm{~mm})$
\end{tabular}


Tabla 4. Resultados del PPD, exámenes serológicos, diagnóstico final, tratamiento y evolución de los casos de fiebre de origen desconocida (FOD) por enfermedad de arañazo de gato (EAG)

\begin{tabular}{ccclll} 
Paciente & PPD & Examen serológico reactivo & Diagnóstico final & Tratamiento & Evolución \\
\hline$\Delta 1$ & 0 & No & EAG & Azitromicina & Curado \\
$\Delta 2$ & 0 & No & EAG & Azitromicina & Curado \\
$\Delta 3$ & 0 & No & EAG, artritis reactiva & No & Mejorado \\
$\Delta 4$ & 0 & No & EAG & No & Curado \\
$\Delta 5$ & 0 & No & EAG & Azitromicina & Curado \\
$\Delta 6$ & 0 & No & EAG & Azitromicina & Curado \\
$\Delta 7$ & 0 & No & EAG, ARJ & No & Mejorado \\
$\Delta 8$ & 0 & No & EAG & Azitromicina & Mejorado \\
$\Delta 9$ & $1 \mathrm{~cm}$ & CMV & EAG, CMV & Doxiciclina & Curado \\
$\Delta 10$ & 0 & No & EAG & Azitromicina & Curado \\
$\Delta 11$ & 0 & HAV & EAG, HAV & Azitromicina & Mejorado \\
$\Delta 12$ & 0 & CMV & EAG, & Azitromicina & Mejorado \\
& & HVS1 & CMV, HVS & & \\
\hline
\end{tabular}

PPD: test de tuberculina, medido en centímetros; $\mathrm{CMV}(+)$, mediante inmunoanálisis quimioluminiscente de micropartículas (CMIA); $\mathrm{HAV}(+)$, mediante la técnica inmunoenzimática de micropartículas (MEIA); HSV1 (+), mediante Elisa.

de FOD. ${ }^{8,9}$ El reservorio natural de $B$. henselae es el gato. Por tanto, se sugiere que, ante un caso de FOD en niños, siempre se debe indagar por la exposición a gatos, buscar lesiones, como cicatrices lineales o pápulas, ocasionadas por el arañazo. En el presente estudio, la mayoría de los casos presentaron cicatrices lineales.

De los exámenes, los reactantes de fase aguda no apoyaron al diagnóstico de infección por $B$. henselae, a diferencia del examen ecográfico, donde se evidenció casos con imágenes redondeadas hipoecoicas en el bazo e imágenes hipoecoicas en el hígado, acompañadas con hepatoesplenomegalia. ${ }^{10,11}$

Los casos estudiados no presentaron antecedentes de comorbilidades; pero, sí coinfección con infecciones virales propias de la edad pediátrica como hepatitis y citomegalovirus.

El caso que presentó hepatitis $A$, caso 11 , presentó aminotransferasas elevadas y serología para hepatitis A IgM reactivo mediante la técnica inmunoenzimática de micropartículas (MEIA), una ecografía con lesiones hipoecoicas en el hígado y en el bazo. No es característico que la EAG presente aminotransferasas elevadas, a diferencia de lo que sucede en la tifoidea y en la infección por brucela.
Hubo coinfección con citomegalovirus en dos casos (9 y 12). En el paciente 9, la reacción fue positiva a la técnica de inmunoanálisis quimioluminiscente de micropartículas (CMIA) con IgM de 2,16 (reactivo positivo > 1). El caso 12 (CMIA IgM 1,3) presentó además herpes simple con lesiones ulcerativas en boca y con serología positivo mediante Elisa IgM de 16,1 (reactivo positivo > 1). Ambos casos presentaron lesiones hipoecoicas en el bazo.

La EAG está relacionada con manifestaciones articulares. En el presente estudio, se encontraron dos casos de artropatías, uno con artritis reumatoide juvenil (caso 3) y otro con artritis reactiva (caso 7). Se debe señalar que otros estudios en busca de la etiología no demostraron la presencia de $B$. henselae en el líquido articular. ${ }^{12,13}$

Un paciente presentó fiebre y convulsión (caso 6), con trastorno del sensorio, disminución de la fuerza muscular, pero con una tomografía cerebral normal, dado de alta como mejorado. Esta manifestación se debe a la capacidad de $B$. henselae de invadir y colonizar las células dendríticas, los eritrocitos, las células endoteliales y la microglía. ${ }^{14}$ Esto es poco frecuente y la mayoría de casos tiene resolución espontánea. ${ }^{15,16}$ Sin embargo, puede haber excepciones y continuar la manifestación neurológica de manera crónica como epilepsia. 
Con respecto al tratamiento, en la mayoría de los casos fue antibioticoterapia empírica, antes de la confirmación serológica, debido a la alta sospecha clínica y epidemiológica. El antibiótico indicado fue la azitromicina, $10 \mathrm{mg} / \mathrm{kg}$ el primer día, seguido de $5 \mathrm{mg} / \mathrm{kg} / \mathrm{d}$, hasta 7 días después de ceder la fiebre. ${ }^{17,18}$

En el presente estudio no hubo casos graves como endocarditis, pericarditis, púrpura trombocitopénica, anemia hemolítica no inmune $o$ angiomatosis bacilar. ${ }^{19-22}$ in embargo, siempre se debe sospechar de un cuadro atípico por $B$. henselae; más aun en un hospital de referencia.

La limitación del estudio está en la dificultad de tenerun examen estándar deoro para el diagnóstico de la EAG. Por tanto, el diagnóstico se realizó mediante la combinación de la epidemiología, la serología, el estudio de imágenes y los exámenes negativos a otra causa.

En los países en desarrollo, con mejoras en su economía, existen factores que condicionan que las enfermedades metaxémicas relacionadas con animales domésticos puedan convertirse en una enfermedad de importancia en salud pública, debido al incremento de la población con familias con hábitos inadecuados en la tenencia de mascotas y de las migraciones. Por estos factores y por los cambios climáticos, ${ }^{23}$ se sugiere la realización de campañas de salud enfocadas en la prevención.

En conclusión, los casos de FOD debidos a infección por $B$. henselae en niños están relacionados con la exposición a gatos, con lesiones hipoecoicas en el bazo y el hígado y con un evolución de la enfermedad con pronóstico bueno.

\section{REFERENCIAS BIBLIOGRÁFICAS}

I. Bass JW, Vincent JM, Person DA. The expanding spectrum of Bartonella infections: Il. Cat-scratch disease. Pediatr Infect Dis J. 1997;16(2):163-179.

2. Fischer GW.The agent of cat scratch disease. In: Mandell G, Douglas $\mathrm{R} \mathrm{Jr}$, Bennett J; eds. Principles and practice of infectious diseases. 3rd Ed. New York: Churchill Livingstone Inc.; 1990.

3. Scott C, Azwa A, Cohen C, McIntyre M, Desmond N. Cat scratch disease: a diagnostic conundrum. Int J STD AIDS. 2009;20:585-586.

4. Huarcaya E, Maguiña C, Merello J, Cok J, Birtles R, Infante B, et al.A prospective study of cat-scratch disease in Lima-Peru. Rev Inst Med Trop Sao Paulo. 2002;44(6):325-330.

5. Jacobs RF, Schutze GE. Bartonella henselae as a cause of prolonged fever and fever of unknown origin in children. Clin Infect Dis. 1998; 26:80-84.

6. Chow A, Robinson JL. Fever of unknown origin in children: a systematic review. World J Pediatr. 20I I;7(I):5-10.

7. Margileth AM, Wear DJ, English CK. Systemic cat scratch disease: report of 23 patients with prolonged or recurrent severe bacterial infection.J Infect Dis. 1987;155(3):390-402.

8. Candela J, Díaz J. Fiebre prolongada como presentación atípica de enfermedad de arañazo de gato en niños. En: XXIII Congreso peruano de Pediatría, Trujillo, 12 al I5 de octubre de 2004.

9. Cerdán S, Candela J. Características clínico epidemiológicas de niños con fiebre de origen desconocido hospitalizados en el INSN. Lima, Perú. 2006 al 2010. [Tesis Segunda Especialización]. Lima: Universidad Nacional Mayor de San Marcos; 2013.

10. Dunn MW, Berkowitz FE, et al. Hepatosplenic cat-scratch disease and abdominal pain. Pediatr Infect Dis J. 1997; 16:269-272.

I I. Bances-Gonzales M, Landa-Garrido L,Amoretti M, Candela-Herrera J. Detección ecográfica de lesiones hepatoesplénicas en niños con enfermedad por arañazo de gato. Rev Per Radiol. 20I I; 15:32-36.

12. Giladi M, Maman E, Paran D, Bickels J, Comaneshter D, Avidor B, et al. Cat-scratch disease-associated arthropathy. Arthritis Rheum. 2005;52:36II-36I7.

13. Dillon B, Cagney M, Manolios N, Iredell JR. Failure to detect Bartonella henselae infection in synovial fluid from sufferers of chronic arthritis. Rheumatol Int. 2000; 19:219-222.

14. Dehio C. Bartonella-host-cell interactions and vascular tumour formation. Nat Rev Microbiol. 2005;3:621-631.

15. Breitschwerdt EB, Maggi RG, Nicholson WL, Cherry NA, Woods $\mathrm{CW}$. Bartonella sp. bacteremia in patients with neurological and neurocognitive dysfunction.J Clin Microbiol. 2008;46:2856-286I.

16. Seah AB, Azran MS, Rucker JC, Biousse V, Martin DF, Newman NJ. Magnetic resonance imaging abnormalities in cat-scratch disease encephalopathy.J Neuroophthalmol. 2003;23(I):16-2I.

17. Florin TA, Zaoutis TE, Zaoutis LB. Beyond cat scratch disease: widening spectrum of Bartonella henselae infection. Pediatrics. 2008; I2I(5):el4I3-1425.

18. Conrad DA. Treatment of cat-scratch disease. Curr Opin Pediatr. 200I;I3(I):56-9.

19. Albini TA, Lakhanpal RR, Foroozan R, Holz ER. Macular hole in cat scratch disease.Am J Ophthalmol. 2005; I40(I):I49-I5I.

20. Koranyi K. Fever, back pain and pleural effusion in a four-year-old boy. Pediatr Infect Dis J. 1994;13(7):657, 72.

21. Rodrick D, Dillon B, Dexter M, Nicholson I, Marcel S, Dickeson $D$, et al. Culture-negative endocarditis due to Houston Complex Bartonella henselae acquired in Noumea, New Caledonia. J Clin Microbiol. 2004;42(4): 1846-1848.

22. Bakker RC, van Heukelem $H$, van de Sandt MM, Bergmans AM. [Visceral granulomas and pericardial effusion caused by a Bartonella henselae infection]. Ned Tijdschr Geneeskd. 1997; I4I (8):388-390.

23. Jameson P, Greene C, Regnery R, Dryden M, Marks A, Brown J, et al. Prevalence of Bartonella henselae antibodies in pet cats throughout regions of North America.J Infect Dis. 1995; I72: I |45- I 49. 